\title{
Structurally perturbed chiral Bragg reflectors for elliptically polarized light
}

\author{
Ian Hodgkinson, Qi Hong Wu, Lakshman De Silva, and Matthew Arnold \\ Department of Physics, University of Otago, P.O. Box 56, Dunedin, New Zealand \\ Akhlesh Lakhtakia and Martin McCall \\ Department of Physics, Imperial College, Prince Consort Road, London SW7 2BZ, UK
}

\begin{abstract}
Received April 26, 2005; revised manuscript received June 17, 2005; accepted June 20, 2005
The structure of an inorganic chiral medium represented as a stack of identical form-birefringent layers that twist steadily with increasing thickness is perturbed by realigning a fraction of each layer to a fixed direction. Experimental results show that the resulting chiral-birefringent composite medium exhibits Bragg resonance with elliptically polarized light, and simulations indicate that Bragg reflectors can be designed for any polarization including linear. (C) 2005 Optical Society of America

OCIS codes: $160.1190,260.1440,310.1620,310.1860$.
\end{abstract}

A resurgence of interest in inorganic structurally chiral media ${ }^{1,2}$ is in turn leading to refinement of deposition techniques. A current focus is the development of complex substrate motion algorithms for maintaining the integrity of spiral nanostructures as they grow in vacuum ${ }^{3}$ or for the direct synthesis of new handed materials. In this Letter we report the deposition of a chiral-birefringent composite material that exhibits Bragg resonance with light of elliptical polarization (EP). We show, via simulations, that the Bragg resonance can be nanoengineered for any elliptical polarization, including the special cases of circular polarization (CP) and linear polarization (LP). Chiral-birefringent structures are common in nature, ${ }^{4,5}$ and the work that we report here was inspired by our observations of overlapping chiral structures in the New Zealand native manuka beetle.

In Fig. 1(a) one dielectric period of a standard chiral material for CP light is shown schematically as a stack of identical birefringent layers that twist steadily with increasing thickness. ${ }^{2}$ Each layer has physical thickness $d$, and the dielectric period, which is the repeat distance for the dielectric properties, has physical thickness $\Omega$. The in-plane principal refractive indices of the layer are $n_{2}$ and $n_{3}$. The fast axis, which is parallel to axis 2 , is marked on the individual layers of the chiral stack. The Bragg resonant wavelength for CP light is $\lambda_{\mathrm{Br}}=2 n_{\mathrm{av}} \Omega$, where $n_{\mathrm{av}}=\left(n_{2}+n_{3}\right) / 2$ is the average of the in-plane refractive indices. In Fig. 1(b) the standard structure has been perturbed by a realignment of part of each layer to a fixed direction. In the new structure the sublayer thicknesses are $d_{A}=f_{A} d$ and $d_{B}=f_{B} d$, where $f_{A}$ is the fraction of the chiral medium $A$ and $f_{B}=1-f_{A}$ is the fraction of the birefringent material $B$. The new structure (b) has the same average refractive index and the same dielectric period as structure (a), and hence the same Bragg wavelength. However, whereas the layer axes of structure (a) are uniformly twisted as is appropriate for resonance with CP light, medium $B$ imposes a pair of fixed axes upon structure (b). The intended optical effect is Bragg resonance for EP light rather than CP light.
The apparatus that we used for computercontrolled serial bideposition ${ }^{2}$ of test layers of inorganic chiral-birefringent composite material in vacuum is shown schematically in Fig. 2. A formbirefringent sublayer of material $A$, for example, is grown by depositing thickness $d_{A} / 2$ at fixed vapor angle $\theta_{v}$ and azimuthal angle $\xi$ followed by the same thickness at azimuthal angle $\xi+\pi$. Such a sublayer has principal axis 1 perpendicular to the substrate, axis 2 (the fast axis) in the deposition plane and parallel to the substrate, and axis 3 (the slow axis) perpendicular to the deposition plane. Composite chiralbirefringent materials emulating the structure that is illustrated in Fig. 1(b) were fabricated by engaging the angular sequence for $\xi$,

$$
0, \pi ; 0, \pi ; \Delta \xi, \Delta \xi+\pi ; 0, \pi ; 2 \Delta \xi, 2 \Delta \xi+\pi ; 0, \pi ; \ldots,
$$

and depositing the appropriate thicknesses.

Figure 3 illustrates the fiber-optic reflectometer that we used for measuring the copolarized reflectance of chiral-birefringent composite coatings. The fixed Fresnel rhomb provides a phase retardation of

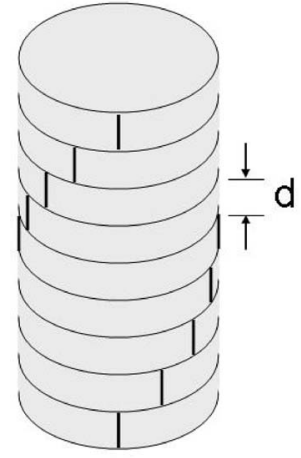

(a)

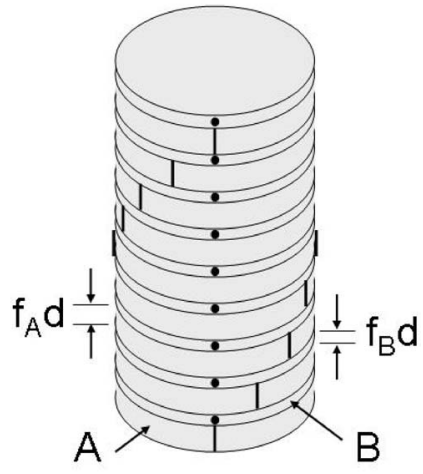

(b)
Fig. 1. One dielectric period of (a) a standard chiral structure represented as a twisted stack of birefringent layers and (b) a chiral-birefringent composite material with the same Bragg wavelength. The bars and dots mark the fast axes of the layers and sublayers, respectively. 


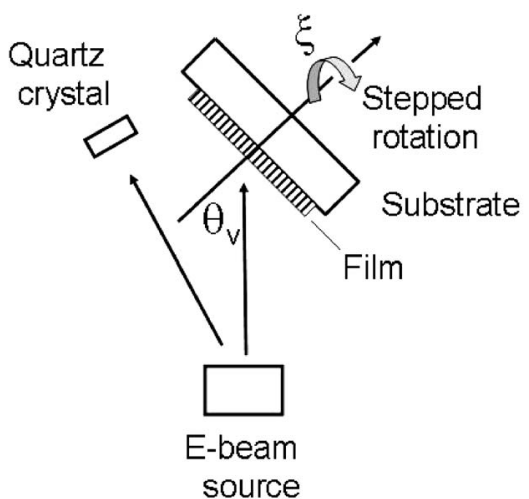

Fig. 2. Apparatus for depositing chiral-birefringent composite materials.

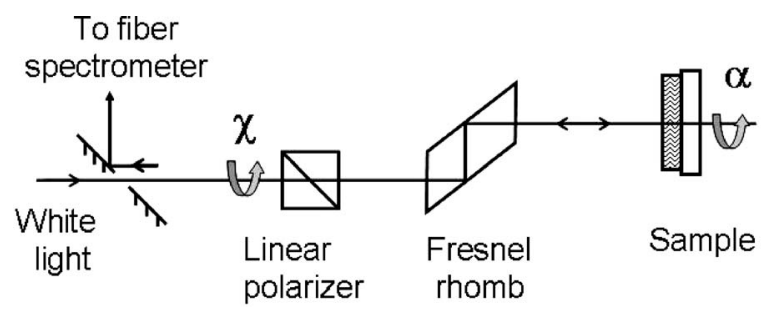

Fig. 3. Apparatus for recording the copolarized reflectance of chiral-birefringent composite coatings.

$\pi / 2$, independent of visible wavelength, ${ }^{6}$ between the horizontal and vertical components of the incident light transmitted by the linear polarizer. It follows that the combination of the linear polarizer and the Fresnel rhomb acts as an elliptical polarizer for the incident light and as an analyzer set to the same elliptical state for the reflected light. By adjusting the angle of the polarizer within the range $-\pi / 4 \leqslant \chi$ $\leqslant \pi / 4$ of auxiliary angle $\chi$, and the angle of the sample within the range $-\pi / 2 \leqslant \alpha \leqslant \pi / 2$ of azimuthal angle $\alpha$, we can determine the copolarized reflectance experimentally for all independent polarizations. ${ }^{6}$ Note that (i) $\chi= \pm \tan ^{-1}(b / a)$, where $a$ and $b$ are the major and minor axes of the polarization ellipse, respectively, $\chi=-\pi / 4$ corresponds to left-handed $\mathrm{CP}$, $-\pi / 4<\chi<0$ to left-handed EP, $\chi=0$ to LP, $0<\chi$ $<\pi / 4$ to right-handed EP, $\chi=\pi / 4$ corresponds to the right-handed CP state, and (ii) $\alpha$ specifies the orientation of the polarization ellipse with respect to the sample.

To illustrate the concept of a Bragg medium optimized for elliptically polarized light we consider a right-handed chiral-birefringent composite film that we fabricated from titania using the following parameters: deposition rate $1 \mathrm{~nm} / \mathrm{s}$, substrate temperature $300^{\circ} \mathrm{C}$, background pressure of oxygen 1.5 $\times 10^{-4}$ Torr, $\theta_{v}=63^{\circ}, \Delta \xi=14.4^{\circ}, f_{B}=0.3, \quad$ sublayer thicknesses chosen to yield a visible Bragg resonance, $N=12$ dielectric periods, cover refractive index $n_{C}=1$, and substrate refractive index $n_{S}=1.52$. Copolarized spectra reflected from the sample, and observed in real time as the polarizer and the sample were rotated manually, revealed strong dependence on $\lambda, \chi$, and $\alpha$. At resonance the Bragg peak appeared as a single peak with sidebands as shown in Fig. 4, and the Bragg wavelength was estimated as
$\lambda_{\mathrm{Br}}=(577 \pm 3) \mathrm{nm}$, and the peak reflectance was estimated as $\approx 0.3$. Following these preliminary observations maps of copolarized reflectance covering all independent polarization states were recorded at the Bragg wavelength (Fig. 5). From a set of eight equivalent maps, recorded during a $2 \pi \times 2 \pi$ scan of the polarizer and the sample, we determined the polarization parameters of the EP Bragg resonance as $\chi_{\mathrm{Br}}=(16 \pm 3)^{\circ}$ and $\alpha_{\mathrm{Br}}=(1 \pm 3)^{\circ}$.

Further values relating to the structure were estimated as follows. A stylus instrument was used to measure the total thickness of the 12-period coating with the result $1975 \mathrm{~nm}$, and hence $\Omega \approx 165 \mathrm{~nm}, d_{A}$ $\approx 9.2 \mathrm{~nm}$, and $d_{B} \approx 4.0 \mathrm{~nm}$. Using the equation $\lambda_{\mathrm{Br}}$ $=2 n_{\text {av }} \Omega$, we obtained $n_{\text {av }} \approx 1.75$, and a simulation of the optical properties, using Berreman $4 \times 4$ matrix algebra, ${ }^{7}$ required $n_{3}-n_{2} \approx 0.07$ to yield the experimental value of the peak reflectance at the Bragg resonance. A plot of normalized copolarized reflectance versus $\chi$ for wavelength $\lambda_{\mathrm{Br}}$ and with the azimuthal angle of the sample set at $\alpha_{\mathrm{Br}}$ is shown for the composite coating in Fig. 6(a). Such a characteristic reflectance profile shows that the EP Bragg resonance is twice as large as the corresponding reflectance with CP light.

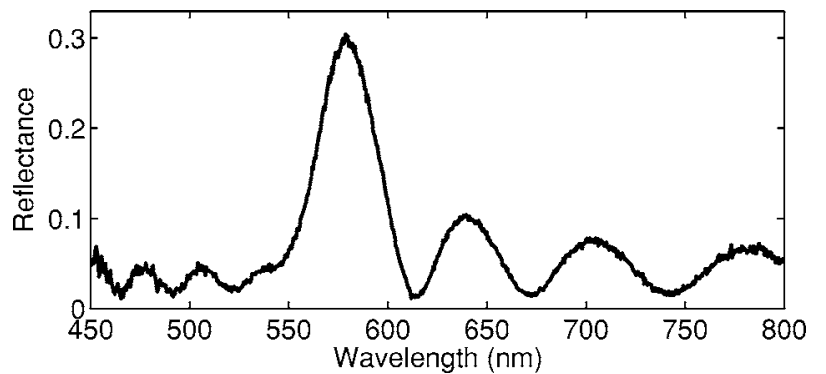

Fig. 4. Copolarized reflectance spectrum recorded for a right-handed birefringent-chiral composite coating with fabrication parameters $\theta_{v}=63^{\circ}, \Delta \xi=14.4^{\circ}, f_{B}=0.3, N=12$, $n_{C}=1$, and $n_{S}=1.52$.

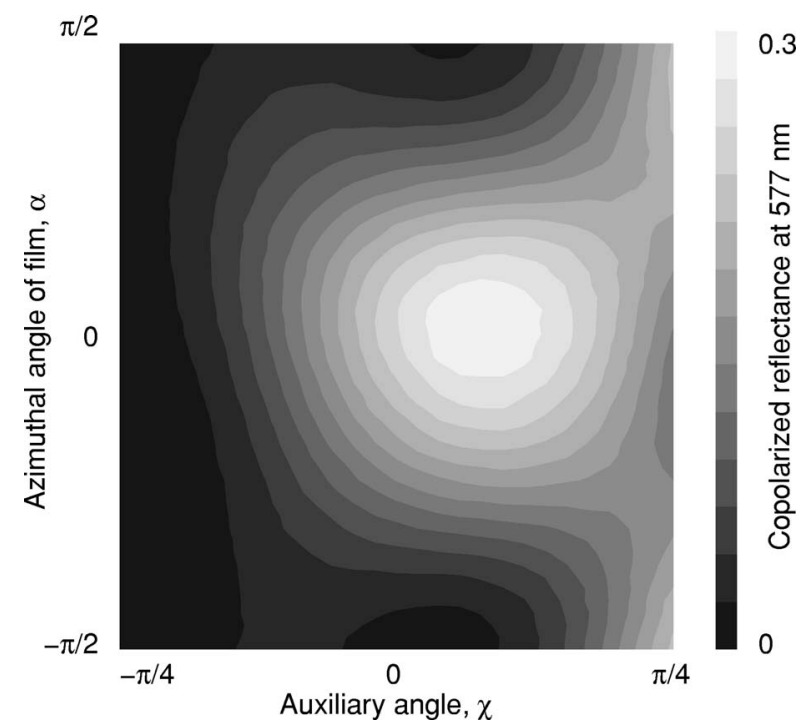

Fig. 5. Copolarized reflectance map recorded for a righthanded chiral-birefringent composite coating with fabrication parameters $\theta_{v}=63^{\circ}, f_{B}=0.3, N=12$, and $n_{C}=1, n_{S}$ $=1.52$. 


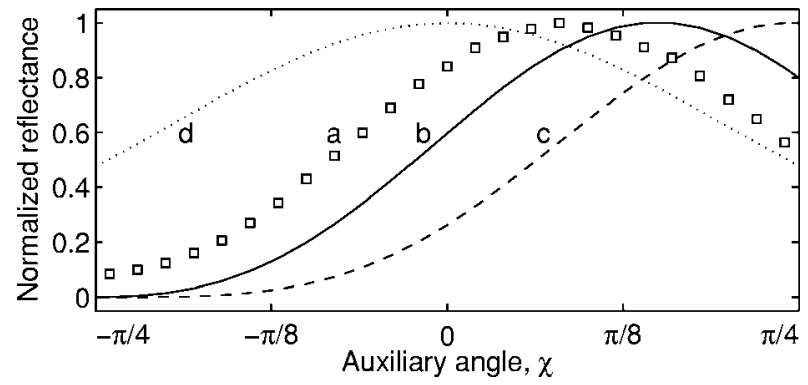

Fig. 6. Normalized copolarized reflectance profiles recorded at $\lambda_{\mathrm{Br}}, \alpha_{\mathrm{Br}}$ for (a) the chiral-birefringent composite coating fabricated with $f_{B}=0.3, N=12$, air and glass bounding media; (b) a simulation of a similar coating with $f_{B}$ $=0, N=12$; (c) an index-matched simulation with $f_{B}=0, N$ $=50$ that positions the Bragg resonance at $\chi=\pi / 4$ (righthanded $\mathrm{CP}$ ); and (d) an index-matched simulation with $f_{B}$ $=0.5, N=50$ that positions the Bragg resonance at $\chi=0$ (LP).

Simulations show that the polarization and strength of the Bragg resonance (from either structure in Fig. 1) may be influenced by spurious reflections associated with mismatch of the refractive indices of the cover, film, and substrate. Relevant to our discussion here a simulation [Fig. 6(b)] with $f_{B}$ $=0, n_{\mathrm{av}}=1.75, n_{3}-n_{2}=0.07, n_{C}=1, n_{S}=1.52, N=12$ yields $\chi_{\mathrm{Br}}=27^{\circ}$, a value that is significantly larger than the experimental value with $f_{B}=0.3$. The perturbed structure in Fig. 1(b), in which the axes of the sublayers of $A$ and $B$ are parallel at the start of the dielectric period, is optimized for maximum peak reflectance and minimum $\left|\chi_{\mathrm{Br}}\right|$ for a given $f_{B}$. In principle spurious reflections can be reduced to negligible levels by index matching or with phase- and amplitude-matched antireflection coatings. ${ }^{2,8}$ In a range of simulations of right-handed composite structures with $n_{3}-n_{2}=0.07, N=50$, and with spurious reflections eliminated by index matching $\left(n_{C}=n_{\mathrm{av}}=n_{S}\right.$ $=1.52$ ) we were able to position the Bragg resonance anywhere within the range $0 \leqslant \chi_{\mathrm{Br}} \leqslant \pi / 4$ by choosing $f_{B}$ from the range $0 \leqslant f_{B} \leqslant 0.5$. Characteristic reflectance profiles simulated for the CP and LP cases are shown in Figs. 6(c) and 6(d).

Additional insight into the perturbed chiral structure can be obtained by expressing it as a sequence of twisted birefringent sublayers $A_{j}$ and aligned birefringent sublayers $B, \ldots B A_{j-1} B A_{j} B A_{j+1} B \ldots$, and then rearranging as a sequence of symmetric Herpin periods with general structure $(B / 2) A_{j}(B / 2)$. Each Herpin period can be replaced by an equivalent sublayer $C_{j}$, with effective principal axes and effective principal refractive indices. ${ }^{7}$ Application of the method to the chiral-birefringent coating that we have discussed in this Letter shows that the equivalent medium $C$ is basically right-handed but with out-of-phase oscillations of approximate amplitude 0.01 on the in-plane effective refractive index values and with an oscillation of approximate amplitude $15^{\circ}$ on the azimuthal angle of the effective fast axis.

In conclusion, we have described a structural perturbation that changes the polarization of Bragg resonance of a chiral reflector from circular to elliptical. In principle any desired polarization, circular, elliptical, or linear, can be nanoengineered for the resonance. The new materials may expand the scope of existing and developing areas where handed materials are employed, such as chiral fiber Bragg gratings ${ }^{9}$ and chiral photonic defect-mode lasers. ${ }^{10}$

Financial support for this project was received from the New Zealand New Economy Research Fund (contract CO8814) and from the MacDiarmid Institute for Advanced Materials and Nanotechnology. Ian Hodgkinson's e-mail address is ijh @physics.otago.ac.nz.

\section{References}

1. A. Lakhtakia and R. Messier, Sculptured Thin Films: Nanoengineered Morphology and Optics, Press Monograph PM143 (SPIE, 2005).

2. Q. H. Wu, I. J. Hodgkinson, and A. Lakhtakia, Opt. Eng. (Bellingham) 39, 1863 (2000).

3. S. R. Kennedy and M. J. Brett, J. Vac. Sci. Technol. B 22, 1184 (2004).

4. A. C. Neville, Biology of Fibrous Composites: Development Beyond the Cell Membrane (Cambridge U. Press, 1993).

5. M. Srinivasarao, Chem. Rev. (Washington, D.C.) 99, 1935 (1999).

6. M. Born and E. Wolf, Principles of Optics (Pergamon, 1980).

7. I. J. Hodgkinson and Q. H. Wu, Birefringent Thin Films and Polarizing Elements (World Scientific, 1998).

8. I. J. Hodgkinson, Q. H. Wu, M. Arnold, M. W. McCall, and A. Lakhtakia, Opt. Commun. 210, 201 (2002).

9. A. Z. Genack, V. I. Kopp, V. M. Churikov, J. Singer, N. Chao, and D. Neugroshl, Proc. SPIE 5508, 57 (2004).

10. J. Schmidtke, W. Stille, and H. Finkelmann, Phys. Rev. Lett. 90, 083902 (2003). 